\title{
RPGOntology: A Semantic Webized Knowledge Base of Radiation Protection Guidelines
}

\author{
Helen Chen, Ph.D. ${ }^{1}$, Jos De Roo, ir. ${ }^{2}$ and Stan Devitt, Ph.D. ${ }^{1}$ \\ ${ }^{1}$ Research and Innovation, Global Architecture and Design Group, Agfa Healthcare, 455 Phillip Street, Waterloo, \\ Ontario, Canada, N2L 3X2 \\ \{helen.chen, stan.devitt\}@agfa.com \\ ${ }^{2}$ Research and Innovation, Global Architecture and Design Group, Agfa Healthcare, Septestraat 27, B-2640 \\ Mortsel, Belgium \\ jos.deroo@agfa.com
}

\begin{abstract}
With the advent of digital imaging technology and the implementation of electronic patient records, the vast amount of information stored in information systems has exceeded any human processing capability. Although this information "overload" consistently pushes the limit of hospital information systems, it provides unprecedented opportunities for decision support to healthcare professionals and patient alike. In healthcare, the information explosion is two-folds: on the one hand, information pertaining to patient healthcare and hospital operation are being added to a hospital's archive. On the other hand, guidelines, protocols and medical research results are generating tens and thousands of entries into medical knowledge bases at an increasing speed. This information is often diverse, with many details, relationships, and sometimes uncertainties. Semantic Web technology provides a universal framework for connecting information semantically and unambiguously. This paper reports our work of building an ontology based on EU Radiation Protection 118 - Referral Guideline for Imaging [1]. Written in RDF [2] and OWL [3], RPGOntology can be consumed by any generic semantic web reasoning engine. This paper also demonstrates how RPGOntology is linked to other existing medical knowledge bases during the reasoning process. Examples using RPGOntology in decision supports are presented, including validation of radiological orders and the recommendation of radiological investigation methods for a given medical condition. Issues regarding clinical validation of RPGOntology and evolving of RPGOntology are also discussed.
\end{abstract}

\section{Introduction}

Radiology is one of those fast-advancing frontiers in modern medicine in the last century. It is especially so with new modalities and visualization technology adding to the repository of radiological investigations treatment methods. The obviously benefit of such radiological procedures is often compromised by the harmful side effects of excessive radiations or is prohibited by the high costs of a certain procedures. Protocols and guidelines are developed to safeguard the patients, as well as to encourage best practices. Radiation Protection 118 - Referral Guideline for Imaging (EU-RPG) [1] published by European Commission presents acceptance criteria among European radiology and nuclear medicine practitioners. Its counterpart in US is a set of Appropriateness Criteria [3] published by the American College of Radiology. Such guidelines are based on community consensus, and often are updated when the volume of new technology or evidence suffices.

Most such guidelines are in free text formats. Table 1 is an excerpt of EU Radiation Protection 118. Given a clinical problem (e.g. Hydrocephalus), a set of investigation methods and their associated radiation dosages are proposed. With regard to each investigation method, a recommendation is made along with the grade indicating the type of available evidence on which the recommendation is based upon. While the correlation between the first three columns seem straightforward, the "comment" column contains discussions, cautions and alternatives of the investigation methods. Reflecting such knowledge truthfully in the ontology can be most challenging. As shown in Table 1, a "comment" can simply state the effectiveness of investigation method (i.e. "Radiography of little use in the absence of focal signs/symptoms") or can suggest alternatives with implication of probabilistic reasoning. A detailed discussion on the conversion of comments in RPGOntology is presented in the next section. 
Table 1. EU Radiation Protection 118 - Referral Guideline for Imaging (Table A excerption)

\begin{tabular}{|c|c|c|c|c|}
\hline CLINICAL PROBLEM & $\begin{array}{r}\text { INVESTIGATION } \\
\{\text { DOSE }\}\end{array}$ & $\begin{array}{c}\text { RECOMMENDATION } \\
\text { \{GRADE\} }\end{array}$ & COMMENT & \\
\hline Headache: chronic & $\begin{array}{l}\text { XR skull, sinus, } \\
\text { C spine (I) }\end{array}$ & $\begin{array}{l}\text { Not indicated } \\
\text { routinely (B) }\end{array}$ & $\begin{array}{l}\text { Radiology of little use in the absence of focal } \\
\text { signs/symptoms. See A13 below }\end{array}$ & \\
\hline $\begin{array}{l}\text { (for children } \\
\text { see Section M) }\end{array}$ & $\begin{array}{l}\text { CT (II) or } \\
\text { MRI (0) }\end{array}$ & $\begin{array}{l}\text { Not indicated } \\
\text { routinely (B) }\end{array}$ & $\begin{array}{l}\text { Some exceptions for specialists or if evidence of raised } \\
\text { intracranial pressure, posterior fossa or other signs }\end{array}$ & \\
\hline $\begin{array}{l}\text { Pituitary and } \\
\text { Juxta-sellar problems }\end{array}$ & MRI (0), & $\begin{array}{l}\text { Specialised } \\
\text { investigation (B) }\end{array}$ & $\begin{array}{l}\text { Demonstration of microadenomas may not be helpful for } \\
\text { management. CT if MRI not available. Urgent referral } \\
\text { when vision deteriorating. Some centers use specific } \\
\text { NM agents. }\end{array}$ & \\
\hline A8 & SXR (I) & $\begin{array}{l}\text { Not indicated } \\
\text { routinely (C) }\end{array}$ & Patients who require investigation need MRI or CT & \\
\hline Posterior fossa signs & MRI (0) & Indicated (A) & $\begin{array}{l}\text { MRI much better than CT. CT images often degraded } \\
\text { by beam hardening artifacts }\end{array}$ & \\
\hline Hydrocephalus & CT (II) & Indicated (B) & $\begin{array}{l}\text { CT adequate for most cases; MRI sometimes necessary } \\
\text { and may be more appropriate in children. } \\
\text { US first choice for infants. NM used in some centres, } \\
\text { especially for shunt function. }\end{array}$ & \\
\hline $\begin{array}{l}\text { (for children see } \\
\text { Section } M \text { ) }\end{array}$ & $X R(0)$ & Indicated (C) & XR can demonstrate whole valve system. & \\
\hline
\end{tabular}

Establishing computable and exchangeable medical guidelines has attracted much attention in the healthcare community [4]. EON [5], Arden Syntax [6], PPRforma [7] are examples of frameworks and languages that can be used to specify computer-interpretable guidelines. Although each has its own merits and user community, it is difficult to share guidelines expressed in different frameworks. Furthermore, due to the procedural nature of most frameworks, the scalability of knowledge bases developed using these frameworks is also of concern given the speed and magnitude of information online. Clinical decision support requires all kinds of information - medical knowledge, guidelines and protocol, patient information, in some cases, information from different domains. For example, the care plan of a patient may be changed if he/she was exposed to anthrax virus by sharing the room with an anthrax patient before the diagnose of this anthrax patient. Here, the geographic layout of a healthcare facility will have to be considered during the reasoning process. Traditional methods meet their limits when dealing with large and heterogeneous knowledge bases on the web.

Developed by W3C community, semantic web technology offers a generic framework to add semantic annotation to webized information. In the world of the semantic web, each concept is uniquely identified via its URI. The open-world assumption of the semantic web allows the reasoning engines to generate new statements based on the facts that can be found in the current knowledge base, while hoping to be able to regenerate the knowledge when new information is captured [8]. This important feature of semantic web technology distinguishes it from other existing medical guideline frameworks that are based on strict closed-world assumption. Semantic Web technology promises the maximum scalability for webized knowledge bases.

In this work, the content of EU-RPG is converted into a webized ontology, namely RPGOntolgy, using W3C's Resource Description Framework (RDF) [2] and Ontology Language (OWL) [3]. For human readability, Notation 3 (N3) is employed during the development of lexicon and axioms in RPGOntology. $\mathrm{N} 3$ is also used for the presentation of rules and RPGOntology in this paper. There are three challenges facing ontology development based on text-based medical guidelines. First, categorization/grouping of recommendations is not consistent. Among 13 tables in EU-RPG text, some tables are named according to anatomical location of the clinical problems (Table A $-\mathrm{H}$ ), some according to the cause of problems (Table $\mathrm{K}$ - Trauma and L - Cancer), and the others according to the type of patients (Table M - Paediatrics and I Obstetricd and Gynaecology). Second, most recommendations are augmented with a paragraph of free text comments. Those comments offer a wealth of options, alternatives, and important notation of an investigation method, but are often non-deterministic. Third, the guidelines will have to be expanded and updated with the arrival of new evidence and imaging technologies. Finally, in order to take full advantage of webized knowledge bases, we are confronted with the challenges of connection - to connect 
RPGOntology with other existing medical ontologies such as SNOMED, GALEN, MESH, NCI ontology, and most likely, knowledge bases developed at specialized institutions.

\section{RPGOntology Lexicon}

The majority of the RPGOntology lexicon can be divided into the following three categories of concepts according to the organization of EU-RPG tables: Clinical Problems, Investigations and Recommendations.

\section{Clinical Problems}

Clinical problems appear in the first column of a table. Figure 1 shows the top-level classes of clinical problems, with each alphabetic letter corresponding to the respective alphabetic letter of a table in EURPG. It is hardly surprising that there may be many different names for a clinical problem due to the regional and institutional differences. Many efforts have been made to uniquely identify medical terms. One notable knowledge base of medical terms is the 'Unified Medical Language Systems" (UMLS) [10], developed by the United States National Library of Medicine. Clinical problems identified in UMLS can be a member of a Clinical Problem Classes in RPGOntology. For example, by stating an RDF triple "umls:C0038454 a rpg:ClinicalProblemA2", the knowledge contains in UMLS can be shared seamlessly with RPGOntology knowledge base during the reasoning process.

It is noteworthy that the mapping of these two knowledge bases does not stop at the syntactic level. The link between umls:C0038454 and rpg:ClinicalProblemA2 can lead a reasoning engine to consider other associated terms and relationships in UMLS. For example, since the more commonly-used names such as "Stroke" and "Apoplexy" belong to the same category of C0038454, the reasoning engine is able to infer the fact that they are also members of rpg:ClinicalProblemA2 class. Therefore the appropriate investigations can be recommended according to EU-RPG. The rules that govern the exchange of knowledge between knowledge bases can be simple, yet the power that comes with the semantically connected knowledge bases is unlimited.

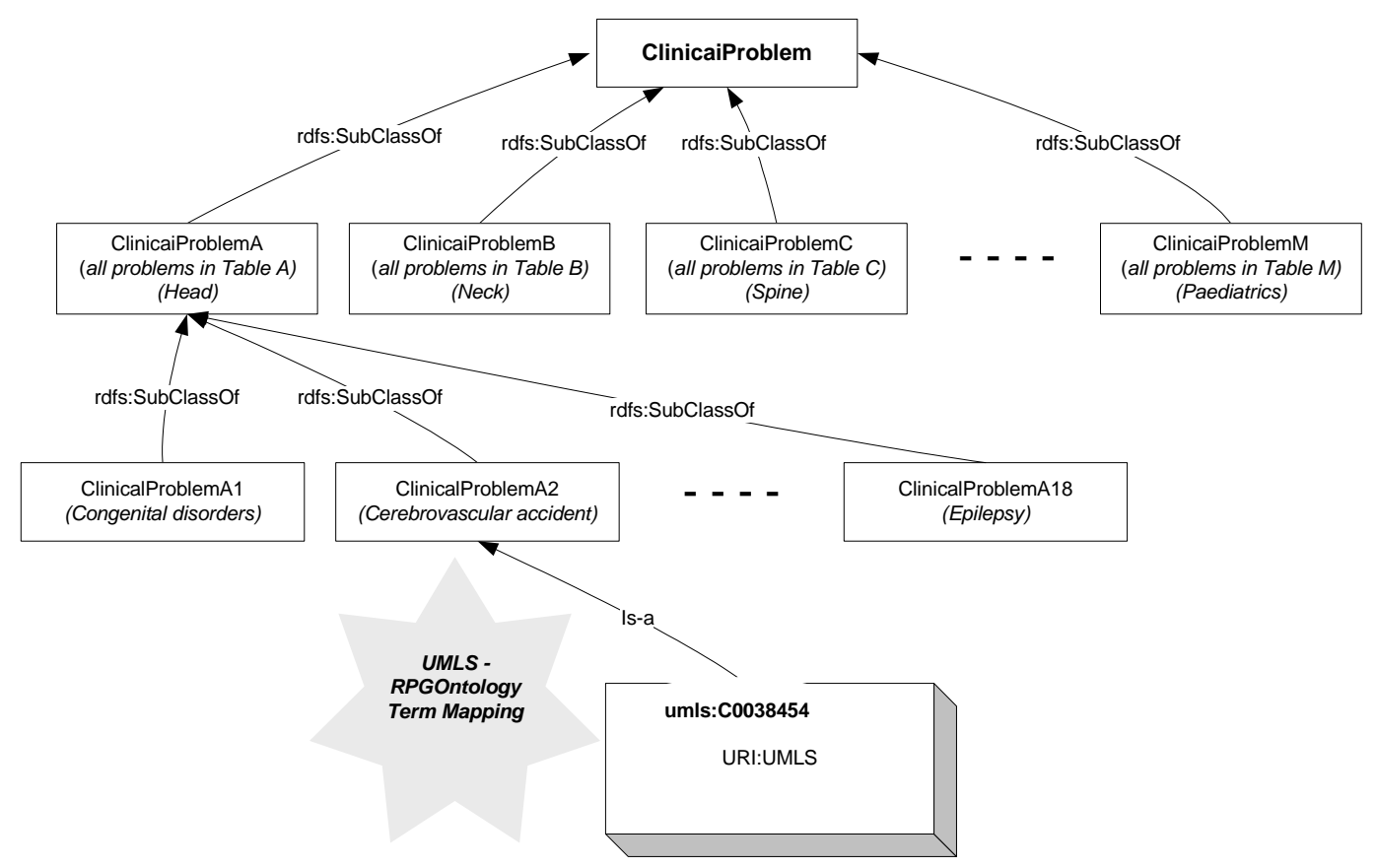

Fig. 1. Top Classes of Clinical Problems 


\section{Investigations}

Investigations are radiological procedures listed in the second column in EU-RPG tables. For a given clinical problem, one or more investigations are recommended. In most cases, logical "OR" is assumed between multiple investigations as in the case of CT and XR for clinical problem A10 (Table 1). A separate identifier is assigned for each investigation method, (i.e. A10_1, A10_2). However, there are some cases where a logical "AND" is specified therefore multiple radiological procedures should be ordered together for a patient, as in the case of Clinical Problem B1 (US and NM are indicated). These investigations are listed as multiple objects for the property rpg:hasInvestigation (see the RPGOntology segment below).

\section{Recommendations}

Recommendations are listed in the third column of EU-RPG tables, along with the grade of evidence. A "indicated" recommendation is most likely to contribute to the diagnosis and management, while a "not indicated routinely" recommendation emphasizes the limitations of a investigation. Medical conditions or criteria for requesting a "not routinely indicated" are often found in the comments. Those conditions are identified as objects of the property "rpg:applyOnlyTo". The conversion of those conditions is not straightforward as that of the clinical problems. Some conditions are further specification of the same type of clinical problems stated in the first column. Some conditions are dependencies of outcomes from a previous treatment or investigation. For example, in EU-RPG Table K, clinical problem K32 (sharp or potentially poisonous swallowed FB) has investigation CXR that is not indicated routinely "unless AXR negative". This condition is reflected in the triple "prg:K32_2 prg:applyOnlyTo AXRNegative". It is not expected to always find direct mapping between those concepts and UMLS. Further denotation can be added to specify desired behaviours or meaning associated with those concepts.

Investigations, recommendations and clinical problems are associated via a set of triples. The following is a segment of RPGOntology (Table B, B1) in N3:

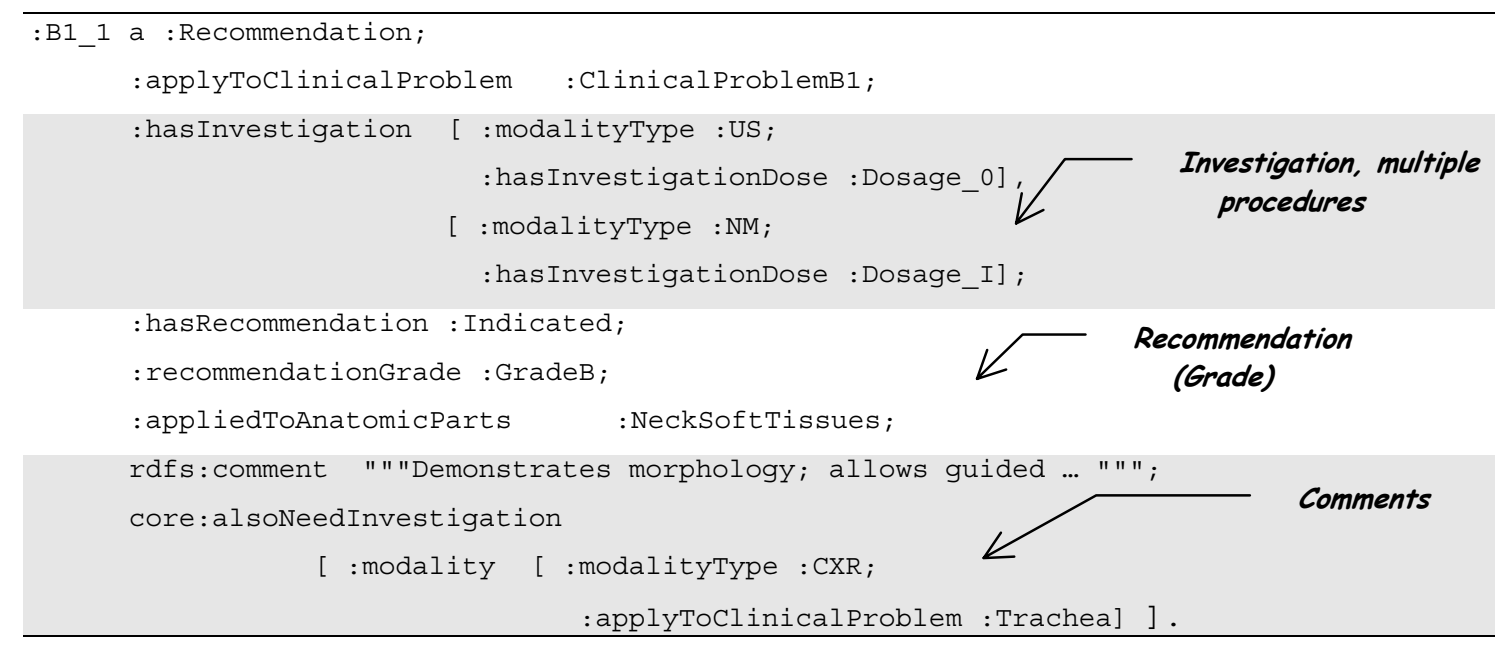

\section{Comments and Alternative Investigations}

Comments often consist of important considerations and alternative investigation methods that must be presented to physicians, as well as reasoning processes. However, the statements are often not definitive. In RPGOntology, comments are first retained as the same text as in EU-RPG. Their meanings are further abstracted into triples. Table 2 lists the properties denoting those meanings. Inevitably not all concepts derived from comments will map to concepts in UMLS or other established medical terminology bases. As stated before, these concepts and meanings are subjected to clinical validation and further denotation. 
Table 2. Concepts Derived from Comments and Intended Meanings

\begin{tabular}{|l|l|l|}
\hline \multicolumn{1}{|c|}{ RPGOntology Concept } & \multicolumn{1}{|c|}{ Meaning } & \multicolumn{1}{|c|}{ Exmaples in EU-RPG } \\
\hline mayRequireInvestigation & Express concept of "likely, possible" & $\begin{array}{l}\text { US valuable for detecting haematoma and } \\
\text { possible injury to some organs, e.g. spleen, } \\
\text { liver. CT may be needed (K38) }\end{array}$ \\
\hline alternativeInvestigation & $\begin{array}{l}\text { Propose an alternative of investigation with } \\
\text { additional criteria }\end{array}$ & $\begin{array}{l}\text { MRI useful when XR normal, especially in high } \\
\text { risk patients. NM and CT can also provide } \\
\text { information here (D20) }\end{array}$ \\
\hline considerInvestigation & $\begin{array}{l}\text { Propose an alternative of investigation under a } \\
\text { certain condition is true }\end{array}$ & $\begin{array}{l}\text { Consider NM for possible metastatic } \\
\text { lesions(C5) }\end{array}$ \\
\hline SeeAlso & Two medical problems might be related & CT may be needed (see K40-K42) (K38)) \\
\hline alsoNeedInvestigation & Express complementary investigation means & $\begin{array}{l}\text { Patients who require investigation need MRI or } \\
\text { CT. (A8) }\end{array}$ \\
\hline BetterThan & $\begin{array}{l}\text { Express one investigation is more preferrable } \\
\text { than the other, equivelent to "more sensitive, } \\
\text { superior, much better than, better than" }\end{array}$ & $\begin{array}{l}\text { MRI much better than CT. CT images often } \\
\text { degraded by beam hardening artefacts.(A9) }\end{array}$ \\
\hline moreSensitiveThan & $\begin{array}{l}\text { Compare effectiveness of two investigation } \\
\text { methods }\end{array}$ & $\begin{array}{l}\text { MRI very sensitive, even limited to spine, pelvis } \\
\text { and proximal femora. (D6) }\end{array}$ \\
\hline
\end{tabular}

\section{Other General Principles in EU-RPG}

Rules and precautions are presented as a general rules for all procedures. For example, in order to protect a foetus from radiation, EU-RPG recommends mothers to avoid radiation at the early stage of their pregnancy. The text in EU-RPG reads: "Woman of reproductive age presenting for an examination in which the primary beam irradiates directly, or by scatter, the pelvic area (essentially any ionizing irradiation between the diaphragm and the knees), or for a procedure involving radioactive isotopes, should be asked whether they are or may be pregnant." RPGOntology has taken such advice into consideration, by explicitly identify the body parts and indication dosage of a recommended investigation. The following rule makes sure that when a investigation is recommended to a woman patient of reproductive age, extra confirmation is carried out. Rules of other policies with regard to resources, costs and frequencies of procedures can also be expressed in a similar fashion.

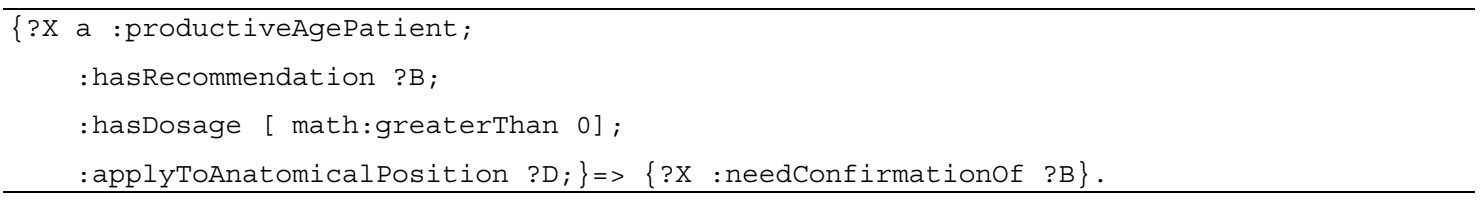

\section{Application of RPGOntology - Reducing Errors and Wastefulness in Radiological Orders}

EU-RPG lists 6 chief causes for errors and wastefulness in radiological procedures: repeating, nonindicative (i.e. no effect on diagnosis or management), too frequent, wrong type, failure to provide critical evidence, and over-irradiation. Some may add wastefulness by performing more expensive investigations where a more economical alternative is equally effective. RPGOntology, connected with other medical knowledge bases and electronic patient records, can effectively alleviate these problems.

When deployed online, each concept in RPGOntology is uniquely identified and accessible via its URI. A generic reasoning engine such as cwm [11] or euler [12] can recommend investigation methods for a given clinical problem at the point of care. It also can perform conformance analysis on radiological procedures in a hospital according to EU-RPG. Rules (in N3) shown here are examples of those used for selecting appropriate investigation methods. Note that in the case of "not indicated routinely", investigations will only be selected when the "extra" condition is satisfied.

Assume a patient has the clinical problem "Salivary Obstruction" (EU-RPG B8). In addition, she also shows "calculus in floor of mouth". Three possible investigations, US, Sialogram (both indicated) and XR (not indicated routinely but the patient problem meets the additional condition) will be recommended. 


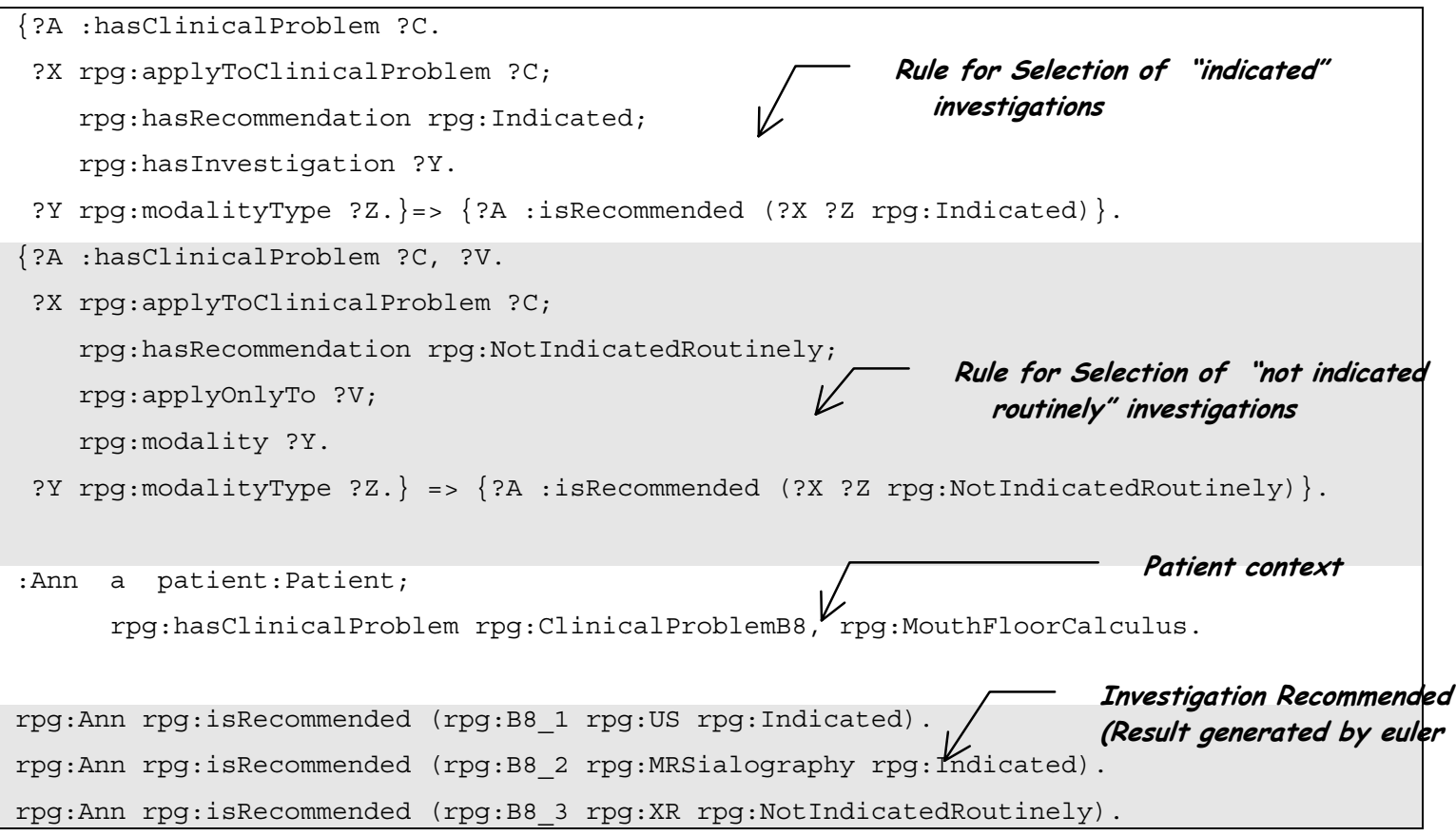

\section{Discussions}

RPGOntology demonstrates how medical knowledge is transformed into semantic webized knowledge base that can be used by semantic web reasoning engines. The ultimate goal is to provide a highly scalable framework for decision support during the diagnosis and treatment processes using proof technology. The risks that may compromise the successful clinical adoption of this technology could be:

- Unstructured order and patient data in a hospital's information systems

- Non-deterministic clauses in guidelines, and to a great extent, in a physician's cognitive and reasoning processes, such as 'likely”, “more effective”, and negation (“will not”, “avoid”)

Although some attributes of an order in a hospital's information system may not be perfectly structured, it is expected that most data in a particular hospital do have some consistency and the variations shall not be overwhelming. A set of queries on the requirement attributes of radiological procedure orders can often provide a good understanding in order to produce a data-mining schema.

RPGOntology made the first step of tackling the "non-deterministic" clauses by expressing them explicitly using a set of concepts (e.g. rpg:mayRequireInvestigation, rpg:moreEffective). Additional resources and facts expressed in triples could be added to constrain the possible interpretations (the possible models). There are active discussions on "reasoning under uncertainty in semantic web", and advancements in this research area will have great impact on decision support in healthcare.

\section{Reference:}

1. European Commission, “Radiation Protection 118 - Referral Guideline for Imaging” (2000) http://europa.eu.int/comm/energy/nuclear/radioprotection/publication/doc/118_en.pdf

2. W3C, "Resource Description Framework (RDF) http://www.w3.org/RDF/

3. W3C, "Web Ontology Language (OWL) http://www.w3.org/2004/OWL/

4. OpenClinical.com http://www.openclinical.org/gmmsummaries.html

5. Stanford Medical Informatics, "EON Project” http://smi-web.stanford.edu/projects/eon/ 
6. HL7, "Arden Syntax for Medical Logic Systems" http://cslxinfmtcs.csmc.edu/hl7/arden/

7. Advanced Computing Lab, "PROForma" http://www.acl.icnet.uk/lab/proforma.html

8. Tim Berners-Lee. "Semantic Web Road Map", http://www.w3.org/DesignIssues/Semantic.html

9. Tim Berners-Lee, "Notation 3 (N3) http://www.w3.org/DesignIssues/Notation3.html

10.United States National Library of Medicine, “Unified Medical Language System” (UMLS) http://www.nlm.nih.gov/research/umls/

11.Tim Berners-Lee “Cwm A General Purpose Data Processor for Semantic Web” http://www.w3.org/2000/10/swap/doc/cwm.html

12.Jos De Roo, "Euler Proof Mechanism" http://www.agfa.com/w3c/euler/ 